\title{
Study of Codon Usage Bias Between Genes of SARS-CoV-2 and Human Tissue-specific Genes
}

\author{
Liheng Lai, Junqi Zhang, Zeyu Chen, Chonghe Sun, Yipeng Zhang \\ International Department of Chengdu Shude High School, No. 398, Bairihong West Street, Chengdu, Sichuan, 670041, China ; \\ renyan@sdgj.com
}

\begin{abstract}
Since December 2019, the SARS-CoV-2 pandemic has posed serious public health threats. Multiple organs in humans are attacked by the virus, but ways and extent to which SARS-CoV-2 affects them vary due to differing t-RNA pools. Codon usage bias of human tissue-specific genes indirectly reflects the cells' t-RNA pool for that specific organ. Once the viral codon usage bias highly matches that of genes expressed in the organ, the virus more effectively utilizes t-RNAs and causes increased damage by reducing translation of human genes. Therefore, finding such a correspondence helps in deducing organs' susceptibility to infection. Using databases and software, we compared codon usage bias of genes in the testes, uterus, brain, liver, ovaries and vulva, and the two longest genes of SARS-CoV-2: ORF1ab and Spike. We found that the two viral genes share 95\% identical codon usage bias. We also found that the liver, ovaries, and testes have the most genes sharing similar codon usage bias to the two viral genes and thus a high possibility for infection. Based on Person's correlation coefficient, we identified the top three genes in the liver, ovaries, and testes. The consequences due to the reduced expression of these genes agree with clinical reports.

KEYWORDS: Microbiology, virology, SARS-CoV-2, tissue-specific genes, codon usage bias, organ's viral susceptibility.
\end{abstract}

\section{- Introduction}

SARS-CoV-2 has been declared a public health emergency of international concern by the World Health Organization. ${ }^{1}$ It is an enveloped, positive-sense RNA virus. The protein spikes of SARS-CoV-2 virus interact with Angiotensin-converting enzyme 2 (ACE2) on the cell membrane to infect cells. ${ }^{2}$ Not only is it expressed in normal lungs, ACE2 is extensively expressed in other areas including smooth muscle cells and the endothelium of vessels in gastrointestinal tracts, brain, liver, gallbladder, kidney, heart, and testes. ${ }^{3,4}$ Thus, it's no surprise that multiple organs in human body are infected by the virus.

Some organs appear to be more susceptible to viral infection than the others. One possible explanation is the codon usage bias of the viral genes and human tissue-specific genes. The genetic code is degenerate. Codons that encode the same amino acid are synonymous codons. However, some codons are used preferentially over the other synonymous codons in many species. This is called codon usage bias. Codon usage bias is measured by relative synonymous codons usage (RSCU). RSCU values below 1 indicate negative codon usage biases and RSCU greater than 1 indicate positive codon usage biases. ${ }^{5}$

The reason for codon bias is still under study. The dominant theory of codon bias for organisms posits that preferred codons correlate to the relative abundances of iso-accepting tRNAs, thereby increasing translational efficiency. ${ }^{6}$ The codon bias may be important for understanding tissue differentiation. Genes selectively expressed in one human tissue can be discriminated from genes expressed in another tissue on the basis of their synonymous codon usage. ${ }^{7}$

Viruses need to hijack the translational machinery of the host cell for a productive infection to occur. They implement tRNA in host cells to produce viral proteins. Viruses tend to share the same codon usage biases as at least one highly expressed protein in the disease targeted area, emphasizing that viral and host codon usage biases are highly correlated. ${ }^{8}$ By studying the codon bias correlation between viral genes and tissue-specific genes, we may be able to determine which tissue a virus is likely to infect as well as which host mRNA would have limited expression.

In this research, we studied Pearson's correlation coefficient of codon bias between coding sequences of one viral gene, ORF1ab, and 169 tissue-specific genes in six organs: liver, brain, testes, ovaries, uterus, and vulva. ORF1ab was chosen because it is the first gene expressed in the host cell. The correspondence between viral and human genes is specifically defined in early viral proteins upon infection as cells undergoing the reprogramming of tRNA pools that favor the translation of late counterparts. ${ }^{9}$ Secondly, ORF1ab is the longest gene, thus its expression can impose great negative effects on the host cell's gene expression. ${ }^{10}$ ORF1ab is translated into $\mathrm{pp} 1 \mathrm{a}$ and $\mathrm{pp} 1 \mathrm{~b}$ proteins. These proteins are cleaved to form 16 non-structural proteins essential for later viral reproduction, viral assembly, immune response modulation, etc. ${ }^{11}$ Our study found that the ORF1ab codon usage bias is $95 \%$ identical to that of the Spike gene, the second largest viral gene. Therefore, only ORF1ab was used to represent the viral results. The liver, brain, testes, ovaries, and uterus tissue-specific genes were chosen because their codon biases show distinctive patterns. ${ }^{7}$

\section{Results and Discussion}

Organs'susceptibility to infection:

The codon usage bias correlation studies in the six chosen organs are shown in Figures 1-6. 


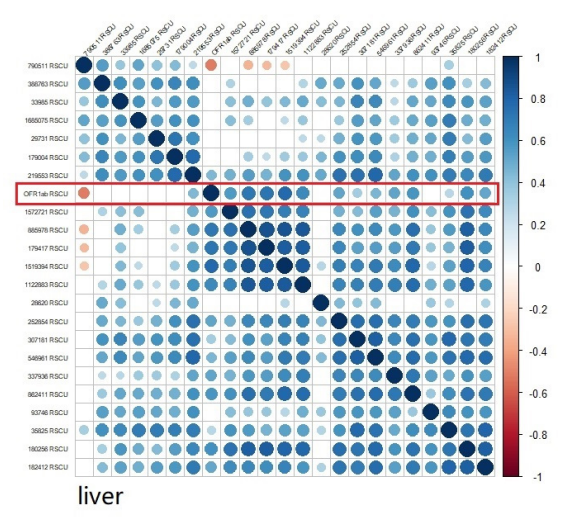

Figure 1: Liver codon usage bias.

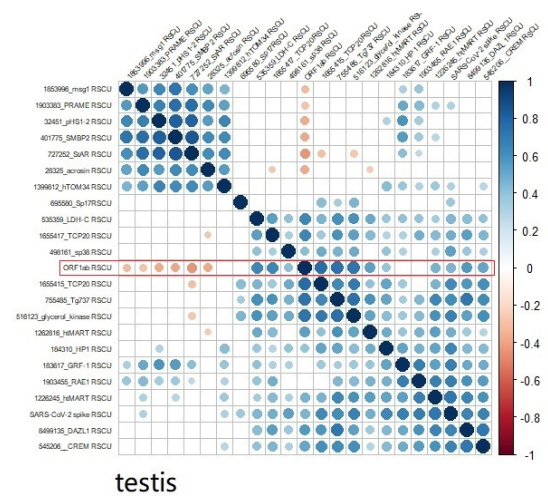

Figure 2: Testis codon usage bias..

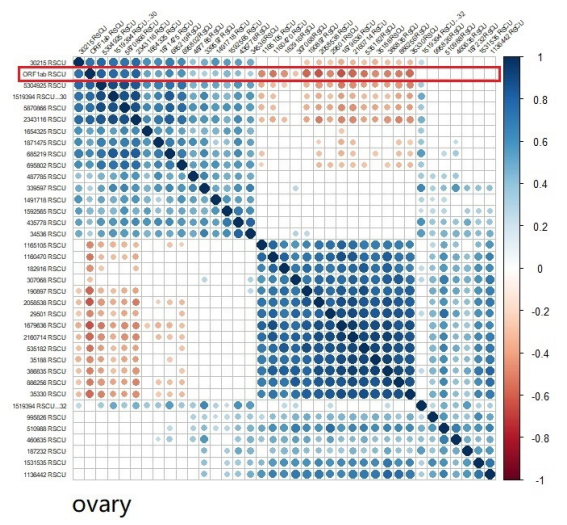

Figure 3: Ovary codon usage bias.

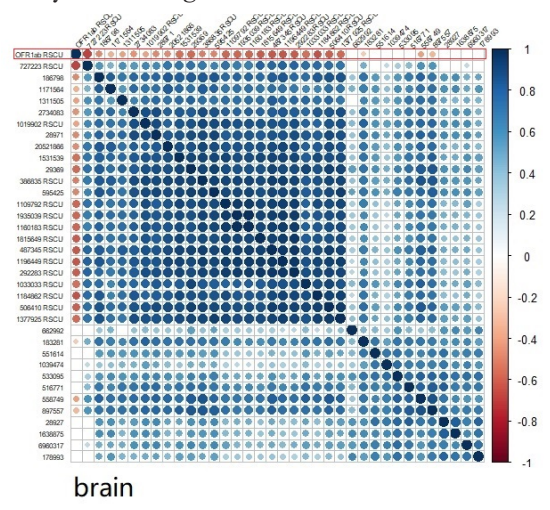

Figure 4: Brain codon usage bias.

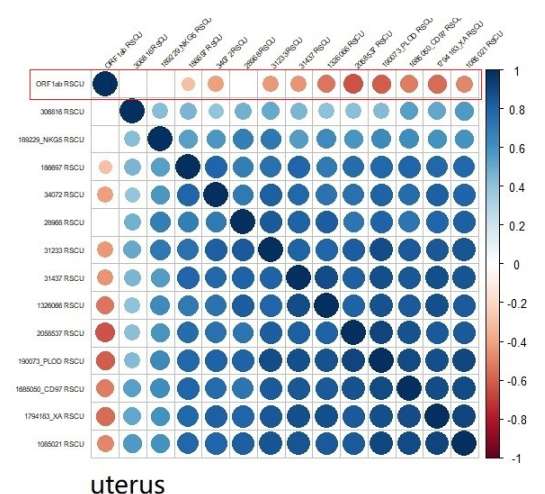

Figure 5: Uterus codon usage bias.

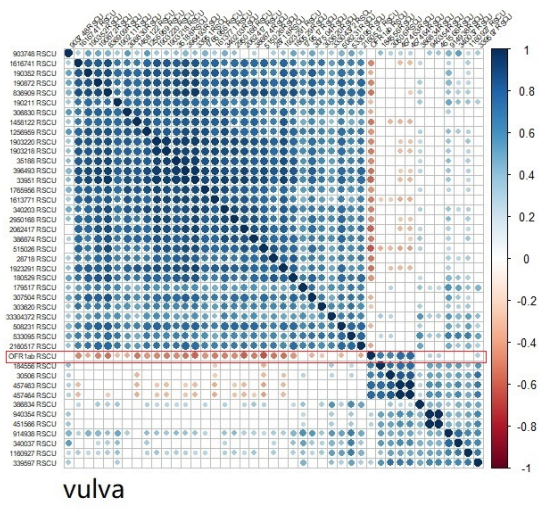

Figure 6: Vulva codon usage bias.

Figure 1-6: The codon usage bias correlation of the viral ORF1ab gene against tissue-specific genes in six organs. The viral ORF1ab gene against tissue-specific genes are in the red box. Dot size is proportional to absolute value of correlation coefficient. The blue and orange color represent positive or negative correlation. The insignificant correlation coefficients are deleted (significance level=0.05).

Our results indicate that the liver, testes, and ovaries have many more genes of significant positive correlation with viral ORF1ab gene in terms of codon bias (Table 1). Because they share similar codon usage bias, the environments in the three organs are suitable for the viral reproduction; these organs possess abundant, correct tRNAs that can be efficiently utilized by the virus for translation. Therefore, they may provide an optimal environment for viral proliferation and, thus, high possibility for infection.

However, in the remaining three organs, vulva, uterus, and brain, all show a strong negative correlation or little to no correlation (Table 1). This indicates an unfavorable environment for viral proliferation. Therefore, even if some viruses do get into those organs via ACE2 receptors, the infection is mild.

We also identified the tissue-specific genes of strongest positive correlation with the viral gene and predicted the damage brought by their reduced expression.

Some of these predictions have manifested in COVID-19 patients (Figure 7). 
Table 1: The number and percentage of genes with significant positive correlations in six organs, the highest correlation coefficient, and ACE2 receptor distribution.

\begin{tabular}{|c|c|c|c|c|c|c|}
\hline Organ & liver & testis & ovary & vulva & brain & uterus \\
\hline $\begin{array}{l}\text { Total number of } \\
\text { tissue-specific genes } \\
\text { studied }\end{array}$ & 22 & 21 & 36 & 42 & 35 & 13 \\
\hline $\begin{array}{l}\text { Number of genes with } \\
\text { significant positive } \\
\text { correlation }\end{array}$ & 14 & 12 & 15 & 7 & 0 & 0 \\
\hline $\begin{array}{l}\text { Percentage of genes } \\
\text { with significant } \\
\text { positive correlation } \\
\text { (\%) }\end{array}$ & 63.64 & 57.14 & 41.67 & 16.67 & 0 & 0 \\
\hline $\begin{array}{l}\text { Highest correlation } \\
\text { coefficient }\end{array}$ & 0.77 & 0.77 & 0.81 & 0.70 & 0 & 0 \\
\hline $\begin{array}{l}\text { ACE2 receptor report } \\
\text { so far }\end{array}$ & Yes $^{12}$ & Yes $^{12}$ & Yes $^{13}$ & No & Yes $^{14}$ & Yes $^{13}$ \\
\hline
\end{tabular}

Percentage of genes with significant positive correlation

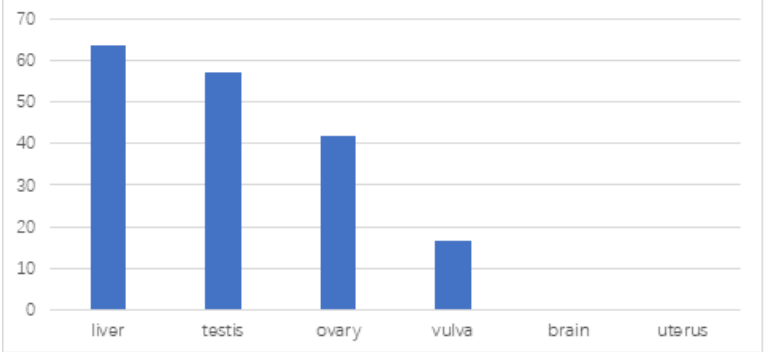

Figure 7: The percentage of genes with significant positive correlation in six organs.

Top 3 tissue-specific genes with highest correlation coefficient with viral gene ORF1ab:

The high positive correlation between the viral and human genes indicates a moderate to strong competition for the same set of tRNA and may reduce the human gene expression.

Liver:

The liver is a multi-functional organ with tasks such as bile production, nutrient storage, lipid and plasma protein synthesis, detoxification, etc.. Based on competition between viral and human genes, we speculate that the lipid metabolism, clotting factor production, and bile excretion may be affected. Additionally, there are case reports supporting the speculation on clotting factor and cholestasis.

Table 2: GI and r-values of different genes in the liver.

\begin{tabular}{|l|l|l|l|}
\hline GI & 1519394 & 179417 & 885978 \\
\hline r value & 0.77 & 0.73 & 0.71 \\
\hline
\end{tabular}

\section{GI 1519394:}

This GI specifies a leptin receptor. The liver is a source of plasma soluble leptin receptors and thus plays a role in modulating total circulating leptin levels and, possibly, its biological activity. One study indicates that plasma soluble leptin receptor levels were significantly associated with a lower risk of type 2 diabetes. ${ }^{15}$ Therefore, reduced expression of leptin receptors in liver may increase the risk of type 2 diabetes. The liver also plays an integral role in regulating lipid metabolism.
Lack of hepatic leptin signaling may result in increased lipid accumulation in the liver and larger, more triglyceride-rich VLDL particles which may contribute to cardiovascular diseases. ${ }^{16}$

GI 179417:

Coagulation factor XIII is activated in the blood coagulation cascade. Plasma factor XIII is a heterotetramer composed of two $\mathrm{A}$ and two $\mathrm{B}$ subunits. $\mathrm{B}$ subunits are produced in the liver. We hypothesize that a reduced expression of coagulation factor XIIIb gene in the liver increases risk of bleeding in COVID-19 patients. In terms of reduced expression, factor XIIIb was significantly decreased in COVID-19 patient sera. ${ }^{17}$ Bleeding symptoms are not frequent in COVID-19, but there was severe bleeding in one COVID-19 patient with congenital bleeding disorders. ${ }^{18}$

\section{GI 885978:}

The SLCO1A2 gene encodes solute carrier organic anion transporter family member 1A2. This protein mediates cellular uptake of organic ions in the liver. We hypothesize that there would be a reduced expression of SLCO1A2 gene in the liver and risk of abnormal delivery of ions in the liver in COVID-19 patients. SLCO1A2 is exclusively expressed on the apical (biliary) membrane of cholangiocytes and may be involved in the reabsorption of substrates that have been excreted into the bile. ${ }^{19}$ Some liver diseases, like cholestasis, are associated with low SLCO1A2 gene expression. ${ }^{20}$ Given the expression of the ACE2 receptor in cholangiocytes, the SARS-CoV-2 may enter these cells, reduce the SLCO1A2 gene expression and thus aggravate cholestasis in patients. ${ }^{21}$ There are reports about association between COVID-19 and cholestasis. ${ }^{22}$

Testes:

The testes are the site of male gametes production and hormone secretion such as testosterone. Here we identify top three genes whose expression may be significantly reduced. All of the three genes are involved in spermatogenesis. The $1^{\text {st }}$ two genes may interact and are implicated in microtubules and microfilaments formation while the third gene may affect energy supply. There are clinical reports showing that the sperm count, and motility is significantly reduced among the affected males. ${ }^{23}$

Table 3: GI and r-values of different genes in the testes.

\begin{tabular}{|l|l|l|l|}
\hline GI & 1655415 & 755485 & 516123 \\
\hline $\mathrm{R}$ value & 0.77 & 0.73 & 0.72 \\
\hline
\end{tabular}

\section{GI 1655415:}

This gene encodes a molecular chaperone that is a member of the chaperonin containing TCP1 complex (CCT). The complex folds several proteins, including actin and tubulin. ${ }^{24}$ With reduced gene expression the CCT complex may malfunction and the ability for cells in testes to fold actin and tubulin will decrease. Since testicular cells are supported by actin microtubule and intermediate filamentbased cytoskeletons to regulate spermatogenesis during the epithelial cycle, ${ }^{25}$ a decrease in actin and tubulin may lead to lower sperm production and infertility among male patients. There are reports showing seminiferous tubular injury among COVID-19 patients. ${ }^{26}$ Therefore, we highly suggest that male 
COVID-19 survivors should be examined for testicular and reproductive function after recovery.

\section{GI 775485:}

The IFT88 gene encodes a member of the tetratrico peptide repeat (TPR) family involved in cilium biogenesis. Testicular cilia play a role in cell signaling and tubular morphogenesis. ${ }^{27} \mathrm{~A}$ low expression level will likely result in weakened cell signaling and impair tubule formation in the testes. Also, IFT88 is essential for the formation of mammalian sperm flagella and mutation results in fewer sperm and shorter flagella. ${ }^{28}$ It's reported that protein product of IFT88 plays a very important role in chaperone function while CCT6B encodes a molecular chaperone. Therefore, a reduced expression of IFT88 genes could affect the protein function of CCT6B.

\section{GI 516123:}

Glycerol kinase 2 (GK2) is a key mitochondrial enzyme for regulating glycerol uptake and metabolism. GK2 is essential for the proper arrangement of crescent-shaped mitochondria to form the mitochondrial sheath during spermatogenesis in mice. ${ }^{29} \mathrm{~A}$ reduced expression of this gene can lead to abnormal localization of mitochondria, causing abnormal mitochondrial sheath formation and leading to exposure of the outer dense fibers and infertility. ${ }^{29}$ Therefore, in COVID-19 patients, the overall spermatogenesis could be affected, and the resulting sperm may show low motility.

Ovary:

The ovaries produce female gametes and secrete hormones such as estrogen and progesterone. Here we identify the top three genes whose expression may be significantly reduced. All three genes are expressed in follicle cells or ubiquitously expressed in the ovaries. They are all involved in follicle and oocyte maturation. While the infection effect on female fertility is controversial, ${ }^{13}$ but our results support a negative impact on female fertility.

Table 4: GI and r-values of different genes in the ovaries.

\begin{tabular}{|l|l|l|l|}
\hline GI & 5304925 & 2343116 & 1519394 \\
\hline$R$ value & 0.81 & 0.81 & 0.77 \\
\hline
\end{tabular}

\section{GI 5304925:}

Zinc finger transcription factors are sequence-specific DNA binding proteins that regulate transcription and affect gene expression. One type of zinc finger transcription factor in follicle cells may regulate follicle maturation and competency for ovulation in Drosophila. ${ }^{30}$ Thus, a reduction of this gene might disturb the normal follicle formation and affect later fertilization.

\section{GI 2343116:}

Karyopherin subunit alpha 5 (KPNA5) protein belongs to the importin alpha protein family and is thought to be involved in nuclear localization signal dependent protein import into the nucleus. Transcripts for KPNA5 are highly abundant in the early pre-vitellogenic stage ovaries, ${ }^{31}$ suggesting that KPNA5, together with other members from importin alpha protein family, may play a significant role in vitellogenesis. Since vitellogenesis occurs during the formation of primary oocytes, a decrease of its expression might lead to underdeveloped primary oocytes and, consequently, unhealthy gametes, which can affect fertility.

\section{GI 1519394:}

Leptin is an important satiety hormone and reproductive regulator. Both leptin and its receptor are found throughout the ovaries. There is evidence indicating possible involvement in oocyte maturation, angiogenesis, follicle rupture, and/or subsequent corpus luteum formation in rats. ${ }^{32}$ Leptin may also have a direct effect on the human ovary. Leptin receptor plays a critical role in the process of ovulation by partially regulating the expression of genes involved in the preovulatory maturation of follicles in human. ${ }^{33}$ Therefore, the presumed reduced leptin in COVID-19 female patients would affect the normal oogenesis and perturb the ovulation.

\section{Significance:}

In this study, a high correlation of codon usage bias was found between SARS-CoV-2 ORF1ab gene and tissuespecific genes of the liver testes and ovaries. This suggests that these three organs are the target of the virus and their function should be checked during examination and reexamination. Our study also predicts the consequence of reduced expression of these human genes whose codon usage bias highly positively correlate with viral ORF1ab gene. Since SARS-CoV-2 is likely to enter those organs and compete with their genes for the tRNAs available for translation, reduced expression of those genes is a plausible prediction. Since ORF1ab is highly similar to the SARS-CoV-2 Spike gene, both genes are likely to have the same effect to the organs at different times during viral development. Thus, the two viral genes are likely to bring the same damage to the organs, making our prediction more significant. It is worth noting that this project only discussed the possible consequences during, not after, viral infection. When the virus is killed by the immune system, there will not be invaders occupying tRNAs originally used by tissue-specific genes; those genes could be expressed normally, and the symptoms could dissipate gradually.

Our investigation provides insights to potential harm of SARS-CoV-2 by studying the organ damage and the affected genes responsible for this. This project also can inform health officials about specific organs that need to be examined to ensure the physical health before and after treatment.

\section{Limitations:}

This research was conducted based on online databases, published papers, and computer operations. It lacks clinical and experimental proof for the views proposed. Also, codon usage bias is sometimes oversimplified without considerations of exceptions. Therefore, future work is needed to demonstrate the validity of our research. What's more, the ongoing mutation of the virus can make the codon usage bias more or less prominent in the future.

\section{- Conclusion}

The liver, testes, and ovaries are three organs that have high correspondence to SARS-CoV-2 in terms of their codon usage bias. They are also the three organs that are most susceptible to SARS-CoV-2 infection. Abnormalities associated with reduced gene expression in the liver includes disturbed reab 
reduced gene expression in the liver includes disturbed reabsorption, bleeding, and disturbed leptin signaling. For testes, symptoms may include lower sperm production, shorter sperm flagella, and lower sperm motility. Male survivors should be examined for testicular and reproductive function after recovery. Possible symptoms for ovary-infected COVID-19 patients include disturbed follicle formation, disturbed normal oogenesis and ovulation, underdeveloped primary oocytes, etc. Our predictions for the liver and testes agree with clinical reports, but the viral effect on female fertility is lacking in clinical reports. ${ }^{20}$

\section{- Methods \\ Sequence:}

The gene IDs for genes expressed in the liver, testes, brain, and uterus is from Tissue-specific codon usage and the expression of human genes. ${ }^{4}$ Complete coding sequences of these genes were obtained from GenBank database (available at: http://www'ncbi-nlm.nih.gov). The full viral genome of SARS-CoV-2 was also obtained from the same source. (GenBank: MN908947.3)

\section{Data analysis:}

The RSCU value of the genes expressed in the chosen organs and viral genome were calculated through CodonW using the formula: ${ }^{2}$

$$
R S C U i j=\frac{X i j}{\frac{i}{n i} \sum_{j=1}^{n i} x j i}
$$

Xij denotes an amino acid's observed number of codons used. ni stands for the amino acid's overall sum of synonymous codons.

CodonW was written by John Peden in the lab of Paul Sharp, Department of Genetics, University of Nottingham.

Codons with an RSCU value greater than 1 are preferred in this gene and vice versa. We deleted three stop codons since they do not code for amino acids. Codons for Trp and Met were also discarded because they each have one corresponding codon and thus have no codon usage bias.

The correlation coefficients between the RSCU value of the genes expressed in different organs and ORF1ab were calculated by R software, using Person's correlation coefficient. A graph of the correlation coefficients ( $r$ ) was obtained. Six graphs were obtained, representing the relationship between the codon usage bias of ORF1ab and the 6 sets of tissue-specific genes from six organs: brain, ovaries, testes, uterus, liver, and vulva.

\section{Prediction:}

We narrowed down our research to the top three tissuespecific genes with positive correlation coefficients. Then we made predictions on the consequences brought by their reduced expression.

\section{Acknowledgements}

We would like to acknowledge Yan Ren, our IB biology teacher, for guiding us through the research process, helping to provide the resources we needed to conduct the research, and sharing helpful feedback on our work. With her guidance, we learned a lot about bioinformatics and improved our under- standing of computer software application for statistical and analytical problems in biology.

\section{References}

1. https://www.who.int/emergencies/diseases/novelcoronavirus-2019/interactive-timeline\#

2. Hoffmann, M.; Kleine-Weber, H.; Schroeder, S.; Kruger, N.; Herrler, T.; Erichsen, S.; Schiergens, T. S.; Herrler, G.; Wu, N. H.; Nitsche, A.; Muller, M. A.; Drosten, C.; Pohlmann, S. SARS-CoV-2 Cell Entry Depends on ACE2 and TMPRSS2 and Is Blocked by a Clinically Proven Protease Inhibitor. Cell 2020, 181(2), 271-280e8.

3. THE HUMAN PROTEIN ATLAS.

4. Hamming, I et al. "Tissue distribution of ACE2 protein, the functional receptor for SARS coronavirus. A first step in understanding SARS pathogenesis."The Journal of pathology vol. 203,2 (2004): 631-7. doi:10.1002/path.1570.

5. Sharp, P. M.; Tuohy, T. M.; Mosurski, K. R. Codon usage in yeast: cluster analysis clearly differentiates highly and lowly expressed genes. Nucleic Acids Res. 1986, 14(13), 5125-43.

6. Sorensen, M. A.; Kurland, C. G.; Pedersen, S. Codon usage determines translation rate in Escherichia coli.J. Mol. Biol. 1989, 207(2), 365-77.

7. Plotkin, J. B.; Robins, H.; Levine, A. J. Tissue-specific codon usage and the expression of human genes. Proc. Natl. Acad. Sci. U. S. A. 2004, 101(34), 12588-91.

8. B. Miller, J.; Hippen, A. A.; M. Wright, S.; Morris, C.; G. Ridge, P. Human viruses have codon usage biases that match highly expressed proteins in the tissues they infect. Biomed. Genet. Genom. 2017, 2(2), 1-5.

9. Hernandez-Alias, X.; Schaefer, M. H.; Serrano, L. Translational adaptation of human viruses to the tissues they infect. bioRxiv, 2020. https://doi.org/10.1101/2020.04.06.027557.

10. Wu, F.; Zhao, S.; Yu, B.; Chen, Y. M.; Wang, W.; Song, Z. G.; Hu, Y.; Tao, Z. W.; Tian, J. H.; Pei, Y.Y.; Yuan, M. L.; Zhang, Y. L.; Dai, F. H.; Liu, Y.; Wang, Q. M.; Zheng, J. J.; Xu, L.; Holmes, E. C.; Zhang, Y.Z. A new coronavirus associated with human respiratory disease in China. Nature 2020, 579(7798), 265-269..

11. Chan, J. F.; Kok, K. H.; Zhu, Z.; Chu, H.; To, K. K.; Yuan, S.; Yuen, K. Y. Genomic characterization of the 2019 novel human-pathogenic coronavirus isolated from a patient with atypical pneumonia after visiting Wuhan. Emerg. Microbes Infect. 2020, 9(1), 221-236.

12.Li, M. Y.; Li, L.; Zhang, Y.; Wang, X. S. Expression of the SARS-CoV-2 cell receptor gene ACE2 in a wide variety of human tissues. Infect. Dis. Poverty 2020, 9(1), 45.

13. Jing, Y.; Run-Qian, L.; Hao-Ran, W.; Hao-Ran, C.; Ya-Bin, L.; Yang, G.; Fei, C. Potential influence of COVID-19/ACE2 on the female reproductive system. Mol. Hum. Reprod. 2020, 26(6), 367-373.

14. Baig, A. M.; Khaleeq, A.; Ali, U.; Syeda, H. Evidence of the COVID-19 virus targeting the CNS: Tissue distribution, host-virus interaction, and proposed neurotropic mechanisms. ACS Chem. Neurosci. 2020, 11(7), 995-998.

15. Qi, S.; Rob, M. V.; James, B. M.; Oscar H. F.; Christos S. M.; Frank B. H. Leptin and soluble leptin receptor levels in plasma and risk of type 2 diabetes in U.S. women: A prospective study. Diabetes 2010, 59(3), 611-8.

16.Huynh, F. K.; Neumann, U. H.; Wang, Y.; Rodrigues, B.; Kieffer, T. J.; Covey, S. D. A role for hepatic leptin signaling in lipid metabolism via altered very low density lipoprotein composition and liver lipase activity in mice. Hepatology 2013, 57(2), 543-54.

17. D'Alessandro, A.; Thomas, T.; Dzieciatkowska, M.; Hill, 
R.; Francis, R.; Hudson, K.; Zimring, J.; Hod, E.; Spitalnik, S.; Hansen, K. Serum proteomics in COVID-19 patients: Altered coagulation and complement status as a function of IL-6 level. J. Proteome Res. 2020, 19(11), 4417-27.

18. Quintavalle, G.; Coppola, A.; Ruggieri, A.; Rivolta, G. F; Fronti, E.; Giangregorio, F.; Michieletti, E.; Tagliaferri, A. Severe bleeding in a patient with factor XIII deficiency and COVID-19. Haemophilia 2020, 0, 1-3.

19. Lee,W.; Glaeser,H.; Smith,L.H.; Roberts, R. L.; Moechel, G. W.; Gervasini, G.; Leake, B. F.; Kim, R. B. Polymorphisms in human organic anion-transporting polypeptide 1A2 (OATP1A2): Implications for altered drug disposition and central nervous system drug entry. J. Biol. Chem., 2005, 280(10), 9610-17.

20.Verena, K.; Martin, B.; Ulrich, W.; Keitel, V.; Burdelski, M.; Warskulat, U.; Kühlkamp, T; Keppler D.; Häussinger, D; Kubitz, R. Expression and localization of hepatobiliary transport proteins in progressive familial intrahepatic cholestasis. Hepatology 2005, 41(5), 1160-72.

21.Zhang,C.; Shi,L.; Wang,F.S. Liver injury in COVID-19: management and challenges. Lancet Gastroenterol. Hepatol. 2020, 5(5), 428-30.

22.Perez, A.; Kogan-Liberman, D.; Sheflin-Findling, S.; Raizner, A.; Ahuja, K. L.; Ovchinsky, N. Presentation of severe acute respiratory syndrome-coronavirus 2 infection as cholestatic jaundice in two healthy adolescents. J. Pediatr. 2020, 226, 278-80.

23. Holtmann, N.; Edimiris, P.; Andree, M.; Doahmen, C.; Baston-Buest Dunja.; Adams, O.; Kruessel, J.; Bielfeld, A.P. Assessment of SARS-CoV-2 in human semen-a cohort study. Fertil. Steril. 2020, 114(2), 233-38.

24. Josefine, V.; Julie, G. The role of the molecular chaperone CCT in protein folding and mediation of cytoskeleton-associated processes: implications for cancer cell biology. Cell Stress Chaperones 2019, 24,17-27

25.Tang, E. I.; Mruk, D. D.; Cheng, C. Y. Regulation of microtubule (MT)-based cytoskeleton in the seminiferous epithelium during spermatogenesis. Semin. Cell Dev. Biol. 2016, 59, 35-45.

26.Yang,M.; Chen,S.; Huang,B.; Zhong, Jing.; Su, H.; Chen, Y.; Cao, Q.; Ma, L.; He, J.; Li, X.; Li, X.; Zhou, Jun.; Fan, J.; Luo, D.; Chang, X.; Arkun, K.; Zhou, M.; Nie, X. Pathological findings in the testes of COVID-19 patients: Clinical implications. European Urology Focus 2020, 6, 1124-29.

27.Dores, C.; Alpaugh, W.; Su, L.; Biernaskie, J.; Dobrinski, I. Primary cilia on porcine testicular somatic cells and their role in hedgehog signaling and tubular morphogenesis in vitro. Cell Tissue Res. 2017, 368(1), 215-23.

28.San Agustin, J. T.; Pazour, G. J.; Witman, G. B. Intraflagellar transport is essential for mammalian spermiogenesis but is absent in mature sperm. Mol. Biol. Cell.2015,26(24), 4358-72.

29.Shimada, K.; Kato, H.; Miyata, H.; Ikawa, M. Glycerol kinase 2 is essential for proper arrangement of crescent-like mitochondria to form the mitochondrial sheath during mouse spermatogenesis. J. Reprod. 2019, 65(2), 155-162.

30.Deady, L.D.; Li, W.; Sun, J. The zinc-finger transcription factor Hindsight regulates ovulation competency of Drosophila follicles. eLife 2017, 6, e29887.

31.Ma, H.; Rexroad, C.; Yao, J. Molecular characterization and expression analysis of importin "a" family genes in rainbow trout. Plant and Animal Genome Conference 2010, 248175.

32.Ryan, N. K.; Van der Hoek, K. H.; Robertson, S. A.; Norman, R. J. Leptin and leptin receptor expression in the rat ovary. Endocrinology 2003, 144(11), 5006-13.

33.Dupuis, L.; Schuermann, Y.; Cohen, T.; Siddappa, D.;
Kalaiselvanraja, A.; Pansera, M.; Bordignon, V.; Duggavathi, $\mathrm{R}$. Role of leptin receptors in granulosa cells during ovulation. Reproduction 2014, 147(2), 221-9

\section{Author}

Liheng Lai is a $12^{\text {th }}$ grade student at the International Department of Chengdu Shude High School. A gold medalist in both USABO (\#5 global rank) and BBO, Liheng has an interest in molecular biology, bioinformatics, computational biology, and biomedical engineering. His dream is to become a biology informaticist.

Junqi Zhang is a $12^{\text {th }}$ grade student specializing in chemistry and biology at the International Department of Chengdu Shude High School. Junqi is interested in biochemistry, pharmacy, animal medicine, and wants to be a veterinarian.

Zeyu Cheng is a $12^{\text {th }}$ grade student at the International Department of Chengdu Shude High School. His area of interests includes environmental science and bioinformatics. $\mathrm{He}$ is always working on applying his knowledge to real life. He currently studies biology and chemistry and wants to be a scholar in the field of computer science, statistics, and biology.

Chonghe Sun is a 17-year-old student specializing in biology at the International Department of Chengdu Shude High School. Chonghe is interested in nutritional biology and scientific research and wants to study nutritional science in university.

Yipeng Zhang is a $12^{\text {th }}$ grade student at the International Department of Chengdu Shude High School. She is interested in molecular biology and microbiology. She hopes to do scientific research in medicine in the future. 